\title{
Investigation of Brain Activity During Listening to and Imagining Music
}

\author{
Yusuke Mitani ${ }^{\mathrm{a}}$, Takuya Kubo ${ }^{\mathrm{b}}$, Yuya Chiba ${ }^{\mathrm{c}}$, Yoshiko Maruyama ${ }^{\mathrm{a}}$, Kenji Moriya $^{\mathrm{a},{ }^{*},}$, Masahiro Nakagawa ${ }^{\mathrm{d}}$ \\ aDepartment of Production Systems Engineering, National Institute of Technology, Hakodate college, \\ Tokura-cho 14-1, Hakodate City, Hokkaido, 042-8501, Japan \\ bDepartment of Electronics and Information Engineering, School of Engineering, Hokkaido University, \\ Kita 8, Nishi 5, Kita-ku, Sapporo City, Hokkaido,060-0808, Japan \\ 'Support Center for Engineering Education, National Institute of Technology, Hakodate College, \\ Tokura-cho 14-1, Hakodate City, Hokkaido, 042-8501, Japan \\ dDepartment of science of technology innovation, Nagaoka University of Technology, \\ Kamitomiokamachi 1603-1, Nagaoka City, Niigata, 940-2188, Japan \\ *Corresponding Author: moriya@ hakodate-ct.ac.jp
}

\begin{abstract}
Effects of imagining music as the relaxation task after the study task compared with listening to music were investigated in terms of prefrontal activity. Prefrontal activity was determined from changes in oxy-hemoglobin $\mathrm{Hb}$ and deoxy-Hb measured by 10-channel wearable optical topography. In this study, we hypothesized that subjects would relax, or experience reduced mental stress when prefrontal activity became inactive. Accuracy of imagining music estimated by VAS was defined as the imagining-accuracy (IA). Three types of music were used during the relaxation tasks were as follows: (1) uplifting song; (2) relaxing song; (3) classical music. Amount of prefrontal inactivity (relaxation effect) were not significantly different between listening to music and imagining music when the IA value was high(i.e., uplifting song and relaxing song).However, its amount was lower the case of imagining music than the case of listening to music when the IA value was low(i.e., classical music), which indicates imagining music accurately is important factor for the effective relaxation.
\end{abstract}

Keywords: Prefrontal activity, NIRS, Imagining music.

\section{Introduction}

Listening to music is an effective relaxation to ensure better rest ${ }^{(1)}$. Effective relaxation is important to concentrate next task(e.g., study, work). Although we listen to music in daily life, we occasionally imagine music in our head.
Imagining, that is mental imagery has been investigated in sports field. Furthermore, several studies have reported that mental imagery gives a slightly same effect as actual training ${ }^{(2)}$. Thus, it is assumed that imagining music is an effective relaxation as well as listening to music. we focused on the effect while imagining music and attempted to investigate the difference between listening to music and imagining music. As is well known, the relationship between emotions and brain activity has been investigated by electroencephalogram ${ }^{\text {(e.g.3,4) }}$. More recently, blood flow of the frontal lobe has been determined using near infrared spectroscopy (NIRS), representing a comparatively simple method of measuring brain activity ${ }^{(e . g .5)}$. Therefore, we attempted to investigate the difference in brain activity between listening to music and imagining music as the relaxation task after the study task in terms of changes in blood flow.

\section{Experimental Methods}

\subsection{Experimental Procedure}

We quantitatively evaluated brain activity under the relaxation tasks (that is, listening to music and imaging music) after the study task. Instead of the study task, subjects were given the mental calculation such as Figure 1. Three tasks were selected for the relaxation tasks: (1) listening to music; (2) imagining music; (3) resting (as control); three types of music were selected when listening to music and imagining music: (1) uplifting song (selected by each subject); (2) relaxing song (selected by each 


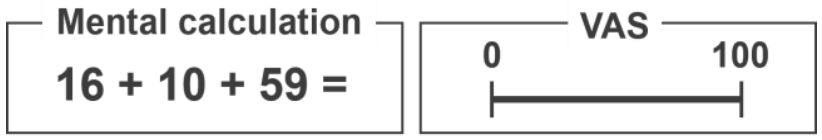

Fig. 1. Example of mental calculation and VAS.

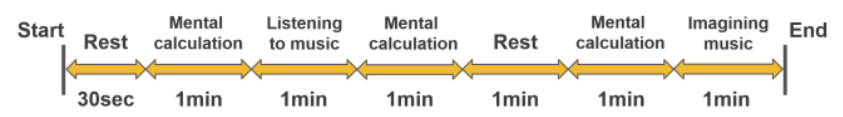

Fig. 2. Experimental procedure.

subject); (3) classical music (Johann Pachelbel "Canon"); it seems that uplifting song and relaxing song are easy to imagine because these songs are listened to often by the subjects. Conversely, it seems that classical music is difficult to imagine because the music with no lyrics. However, it cannot be investigated the effect of imagining music if the subjects cannot imagine at all. Therefore, Johann Pachelbel "Canon" which is a famous classical music was selected. Additionally, the accuracy of imagine was estimated from 0 to 100 levels using visual analog scale (VAS) such as Figure 1 after the experiment. Here, we defined this index as the imagining-accuracy (IA). Assuming that 0 is a state which cannot be imagined and 100 is a state which can be completely imagined, the subjects specify how much accurately music could be imagined by indicating a position along a continuous line between two end-points when responding to VAS.

Experimental procedure was shown in Figure 2. The first $30 \mathrm{sec}$ of the procedure consisted of a rest period. The duration of the study task was $1 \mathrm{~min}$. The duration of each relaxation tasks as $1 \mathrm{~min}$. The subjects then completed the 1-min study task and then conducted the each 1-min relaxation tasks. The subjects repeated this procedure with the different three types of music at adequate time intervals. The experiment procedure so far was conducted three times each on separate days per person.

During experiment, the subjects was sitting on a chair and looking at the PC screen. Instructions of each task and mental calculation problems were displayed on the PC screen. The subjects were instructed to keep their eyes close during relaxation task. Noise-canceling headphone (QC25 noise cancelling headphones, Bose Corporation LLC.,) was used while listening to music.

\subsection{Brain Activity Analysis}

Activity in the prefrontal area was investigated by measuring changes in oxygenated hemoglobin (oxy-Hb) and deoxidized hemoglobin (deoxy-Hb) concentrations determined by 10-channel $(\mathrm{CH})$-wearable optical topography (WOT-100, Hitachi High Technologies Ltd.,) with near-infrared spectroscopy (NIRS) at a sampling frequency of $5 \mathrm{~Hz}$. When near-infrared ray passes through hemoglobin, the intensity of near-infrared ray changes with differences in the absorption spectra of oxy-Hb and deoxy-Hb. By measuring this change, oxy-Hb and deoxy-Hb concentration changes can be calculated. If oxy-Hb increased and deoxy-Hb decreased, this indicated that the corresponding region of the prefrontal area is active compared with the previous condition. When oxy-Hb decreases and deoxy-Hb increases, prefrontal activity is inactive (summarized as Table 1). In this study, if $\mathrm{Hb}$ in the regions surrounding the $\mathrm{CHs}$ involved in the study task changed during the relaxation task as per the above definition of inactivity, we proposed that the subjects were relaxed in the current task compared with previous task.

The correspondence between the measurement region of the prefrontal area and each CHs was shown in Figure 3. In this study, two analyzes were performed: (1) average of $\mathrm{Hb}$ changes in the rest and average of $\mathrm{Hb}$ changes in the study task after the rest were compared to investigate which regions of the prefrontal area become active during the study task; (2) average of $\mathrm{Hb}$ changes in each relaxation tasks after the study task and average of $\mathrm{Hb}$ changes in each study tasks were compared to investigate rates of prefrontal inactivity and amount of prefrontal inactivity during the relaxation task; considering the influence of suddenly change, analyzes were performed except for ten seconds of the beginning and five seconds of the ending in each tasks. The remaining 45 seconds were divided into three sections of 15 seconds and the $\mathrm{Hb}$ changes was analyzed in detail using the respective average value.

Table 1. Brain activities determined from changes of oxy-Hb and deoxy-Hb.

\begin{tabular}{|c|c|c|}
\hline $\begin{array}{c}\text { Change of } \\
\text { oxy-Hb }\end{array}$ & $\begin{array}{c}\text { Change of } \\
\text { deoxy-Hb }\end{array}$ & Brain Activity \\
\hline & $\checkmark$ & Active \\
\hline & $\checkmark$ & Inactive \\
\hline
\end{tabular}



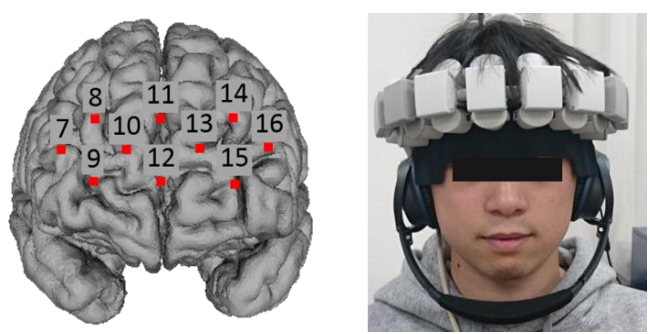

Fig. 3. Measurement channel $(\mathrm{CH})$ in the prefrontal area.

\section{Results and Discussion}

Measurements of blood flow in the prefrontal cortex was conducted in five subjects (healthy male students aged 19-21 years); therefore, 45 data in total were obtained because the experiments were performed three times in three types of music per person. We investigated which regions of the prefrontal area become active during the study task. Figure 4 shows the proportion of each $\mathrm{CH}$ that became active during the study task. The results indicated that active rates in $\mathrm{CH} 9, \mathrm{CH} 12$ and $\mathrm{CH} 15$ of the prefrontal cortices, respectively, were higher than other channels; considering the individual differences among subjects, we focused on prefrontal activity around high activation rate $\mathrm{CHs}$ (i.e., $\mathrm{CHs} 7,9,10,12,13,15$, and 16) when investigating effective relaxation after the study task.

We calculated the changes amount of the $\mathrm{Hb}$ average concentration change in the task compared the $\mathrm{Hb}$ average concentration change in the previous task. Figure 5 shows an example of the changes amount of $\mathrm{Hb}$ average concentration change in $\mathrm{CH} 9$ (Subject A). The vertical axis shows how much $\mathrm{Hb}$ average concentration change in the task changed compared to the previous task. When the brain activity is active, the background is red, and when it is inactive, it is blue. It is indicated that the more the changes

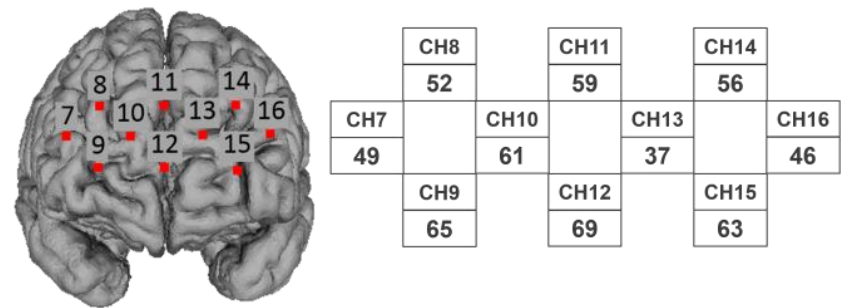

Fig. 4. Activation rates in $\%$ during the study task of each channel $(\mathrm{CH})$ of the prefrontal area. The listed values indicate the rates at which each area became active per total number of measurements $(n=45)$. of the oxy-Hb is low and the more the changes of the deoxy-Hb is high, prefrontal area is more inactive.

We calculated each rates of prefrontal inactivity in three types of music during the three relaxation tasks performed after the study task that are shown in Figure 6. Colored bars represent the three relaxation tasks: listening to music, imagining music, rest. This result shows the tendency of prefrontal inactivity that is easy to become the relaxation state in each relaxation tasks in each music types. The prefrontal area tended to be inactive while the subject was imagining music in comparison with listening to music and rest in uplifting song and relaxing song. However, rates of inactivity were not significantly different among the various relaxation tasks and the various types of music $(\mathrm{P}>0.05)$.

We calculated each average change amount of $\mathrm{Hb}$ average concentration change in three types of music during the three relaxation tasks performed after the study task that are shown in Figure 7. Red bars represent the changes amount of the oxy-Hb and blue bars represent the changes amount of the deoxy-Hb. In this study, assuming that the changes amount of the $\mathrm{Hb}$ average concentration

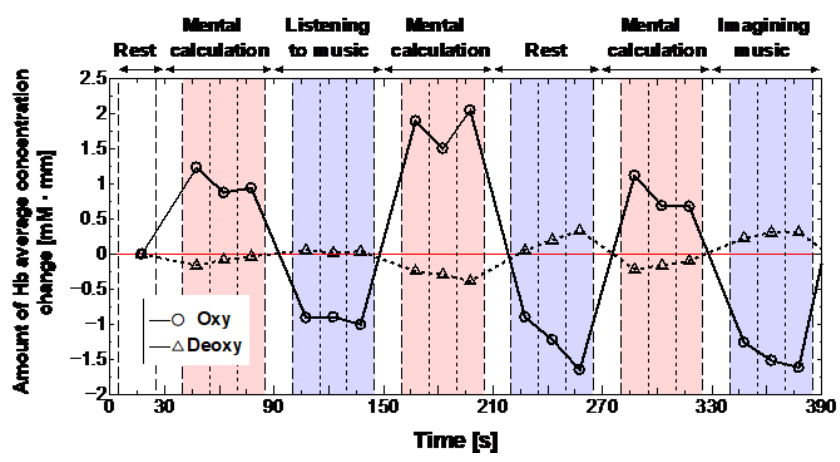

Fig. 5. Subject A: An example of the changes amount of $\mathrm{Hb}$ average concentration change in $\mathrm{CH} 9$.

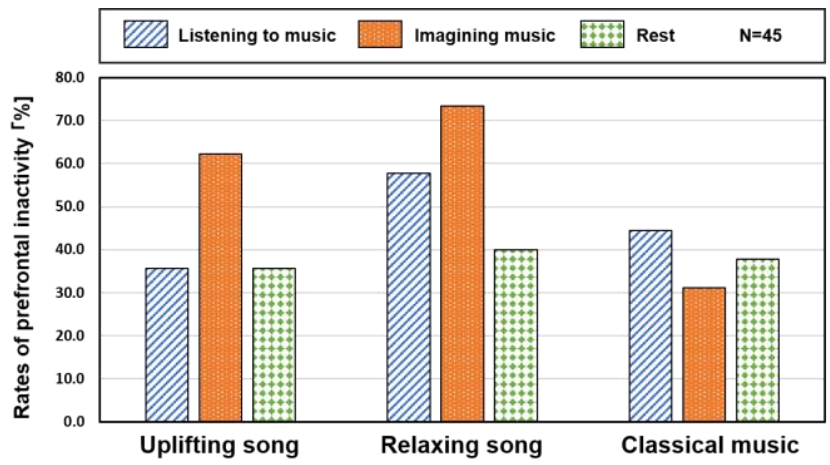

Fig. 6. Rates of prefrontal inactivity in three types of music during the three relaxation tasks after the study task. 
change is amount of prefrontal inactivity (that is, relaxation effect), the addition of the changes amount of oxy-Hb and deoxy-Hb average concentration change was assumed to be the relaxation effect. The relaxation effects were not significantly different among the various relaxation tasks in uplifting song and relaxing song $(\mathrm{P}>0.05)$. However, there was significant difference between listening to music and imagining music in classical music $(\mathrm{P}<0.05)$. The relaxation effect while the subject was listening to music was higher than imagining music in classical music.

Next, we focused on the imagining-accuracy (IA) that is measured by VAS performed after the experiment. Table 2 shows each average value of IA in three types of music. The value of IA in classical music was lower than IA in uplifting song and relaxing song. This means that classical music could not be imagine accurately by the subjects compared with uplifting song and relaxing song. Consequently, it suggested that there was no difference in the relaxation effect between listening to music and imagining music when the IA value was high. Although, its amount was lower the case of imagining music than the case of listening to music when the IA value was low. Therefore, these results indicate that imagining music might have the same effects as the listening to music, if music could be accurately imaged inside head. Additionally, it is believed that if the IA value increases (that is, accustomed to imagining music), the relaxation effect also increases.

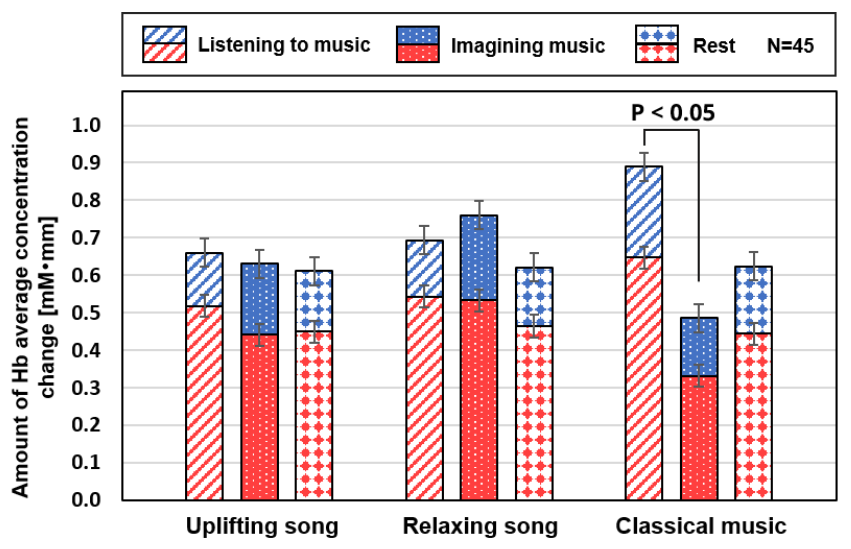

Fig. 7. Average changes amount of $\mathrm{Hb}$ average concentration change in three types of music during the three relaxation tasks after the study task.
Table 2. Average value of IA in three types of music.

\begin{tabular}{cccc}
\hline & Uplifting song & Relaxing song & Classical music \\
\hline IA & 78.6 & 79.0 & 53.4 \\
\hline
\end{tabular}

\section{Conclusions}

We examined the difference in prefrontal activity between listening to music and imagining music as the relaxation task after the study task in terms of changes in blood flow. Prefrontal activity was determined from changes of oxy-Hb and deoxy-Hb concentration. In this study, accuracy of imagining music estimated by VAS was defined as the imagining-accuracy (IA). The mental calculation, which was used as a study task, activated prefrontal activity around $\mathrm{CHs} 9,12$ and 15. Rates of inactivity after the study task in the prefrontal area were not significantly different among the all three relaxation tasks (listening to music, imagining music, and resting) and the three types of music (uplifting song, relaxing song, and classical music). Amount of inactivity (relaxation effect) were not significantly different between listening to music and imagining music when the IA value was high. However, its amount was lower the case of imagining music than the case of listening to music when the IA value was low. this finding indicated that imagining music might have the same effects as the listening to music, if music could be accurately imaged inside head. The effects of imagining music against the various IA value should be investigated in subsequent studies because there is a possibility that IA is an important factor in the relaxation effect. In addition, measuring parasympathetic nervous system activity to support the relaxation effect in prefrontal activity are required. This experiment was conducted in healthy students aged 19-21 years (i.e., young adults). Different results might be found among older people or those from different cultures.

\section{Acknowledgment}

A part of this study was supported by a grant from Nagaoka University of Technology for collaborative research with National Institute of Technology. 


\section{References}

(1) K. Moriya, I. Kurimoto, N. Ezaki, M. Nakagawa : "Influences of Listening to Music in Study Break on Brain Activity and Parasympathetic Nervous System Activity", Journal of the Institute of Industrial Applications Engineers, Vol.6, No.1, pp. 34-38, 2018

(2) BC. Clark, NK. Mahato, M. Nakazawa, TD. Law, and JS. Thomas : "The power of the mind: the cortex as a critical determinant of muscle strength/weakness", J Neurophysiol, 112, pp. 3219-3226, 2014

(3) S. Nishida, T. Hatada and M. Nakagawa : "An Estimation Method of Cognitive States with Fractal Dimensions of EEG", Proceedings of the 11th International Conference on Humans and Computers, pp.195-200, 2008

(4) C. Gelinas, M. Boitor, M. Ranger, C.C. Johnston, M. Marchie, F. Cervero, M. Choiniere : "Is near infrared spec- ' troscopy valid for the detection of procedural pain in postoperative cardiac surgery intensive care unit adults?", Journal of Near Infrared Spectroscopy(online access), 2017

(5) S. Matsushita and M. Nakagawa : "Emotional Information Analysis using Optical Topography", IEIEC Trans. Fundamentals, Vol.J88-A, No.8, pp.994-1001, 2005

(6) S. Akselrod, D. Gordon, F.A. Ubel, D.C. Shannon, A.C. Barger and R.J. Cohen : "Power spectrum analysis of heart rate fluctuation: a quantitative probe of beat-to-beat cardiovascular control", Science, 213, pp.220-222, 1981

(7) Editorial board of "Encyclopedia of Sound" : "Encyclopedia of Sound", Maruzen Publishing, p.33, 2006, ISBN978-4-621-07660-6

(8) K. Moriya, Y. Chiba, H. Yoneta, R. Akiyama and H. Tazawa : "Simultaneous measurement of instantaneous heart rate and breathing activity in newly hatched chicks", Br. Poult. Sci., Vol.44, No.5, pp.761-766, 2003

(9) K. Moriya, R. Akiyama, E.M. Dzialowski, W.W. Burggren and T. Hiroshi : "Development of heart rate circadian rhythm in chickens", Avian and Poultry Biology Reviews, Vol.15, No.3/4, pp.211-218, 2004 